\section{Congenital bicuspid aortic valve: Differential prevalence across different South African population groups}

To the Editor: Congenital bicuspid aortic valve (BAV) is considered the most common congenital cardiac defect, with a reported prevalence in North American and European populations of 0.5 $2 \% .{ }^{[1]}$ Adverse outcomes in BAV are most frequently related to aortic regurgitation, aortopathy with an associated risk of aortic dissection, infective endocarditis and the development of aortic stenosis later in life. ${ }^{[2]}$ To the best of our knowledge, there are no epidemiological studies documenting the prevalence of BAV in southern Africa. We provide the first report of BAV in a screened, healthy South African (SA) population and highlight an unprecedented finding of a differential prevalence of BAV across different SA population groups, with an almost negligible prevalence in black SA children.

Our data are sourced from the Echo in Africa (EIA) project, an echocardiographic rheumatic heart disease (RHD) screening programme based in Western Cape Province, SA. Established in 2014 by SUNHEART (a non-profit organisation linked to the Division of Cardiology at Tygerberg Academic Hospital in Cape Town) and the British Society for Echocardiography, and approved by the Human Research and Ethics Committee of Stellenbosch University (ref. no. N14/04/038), EIA remains the largest and only ongoing large-scale RHD screening programme in SA. Study ethics approval and operational permissions were obtained through Stellenbosch University and the Department of Education in the Western Cape, respectively.

A retrospective review of the EIA database was performed to identify all cases of BAV. BAV was defined as congenital fusion (complete or partial) of two aortic valve cusps, classified according to their leaflet morphology (right-left coronary, right non-coronary and left non-coronary). ${ }^{[2]}$

Of a total of 6171 screened participants (aged 13 - 19 years, mean 15.5 years), 15 study participants $(68 \%$ male) were identified with BAV, yielding an overall prevalence of 2.4 cases/1 000 (95\% confidence interval (CI) 1.5 - 4). Right-left coronary fusion was observed in 9 cases and partial fusion of the left non-coronary cusp in 3 , and there was 1 case each of right non-coronary and left noncoronary cusp fusion. An example of an EIA BAV case with right-left coronary cusp fusion is presented in Fig. 1.

Further classification of BAV cases according to population group yielded surprising results. Our screening cohort comprised three population groups: black, white and mixed South African (an ethnic group of Khoisan-European-African-Malay mixed ancestry). We identified a low prevalence of BAV among mixed-ancestry children ( 1.3 cases $/ 1000 ; 95 \%$ CI 0.5 - 3.4). BAV was an exceedingly rare finding among black SA children, with only 1 case found among 2334 children screened (0.4 cases/1 000; 95\% CI 0.1 - 2.4/1 000). There was a statistically significant difference between the proportion of BAV among black and mixed-ancestry children and that in white children $(p<0.0001)$. White SA children had a BAV prevalence of 12.9 cases/1 000 (95\% CI 7.22 - 22.9), similar to that reported in Northern American and European populations.

The reasons that underpin this notable finding require further study. Instances of reduced prevalence of BAV among African Americans have been documented in single-centre hospital-based registries in the USA, although not to the same degree as described in the present report. ${ }^{[3,4]}$ Potential bias in the selection of the cohorts, reflecting socioeconomic status rather than a specific population group, is mitigated by the fact that BAV is a known congenital rather than acquired condition, so that lifestyle differences are less likely to have an impact. Furthermore, BAV-associated attrition events

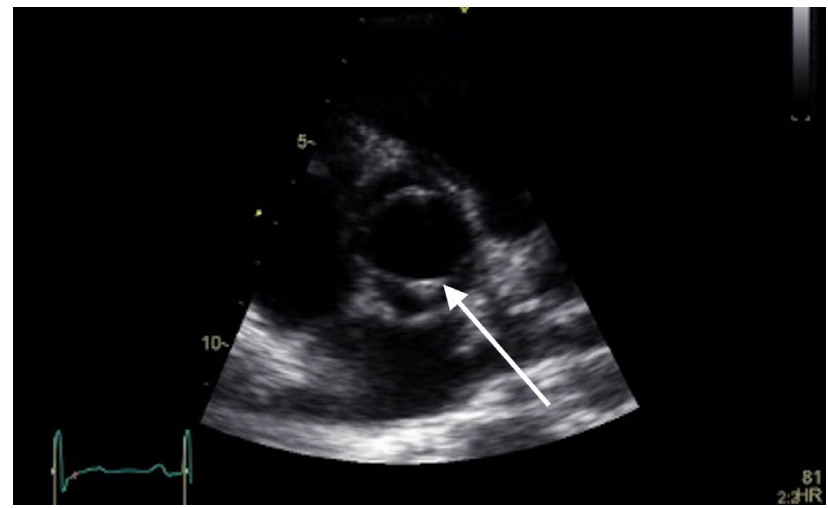

Fig. 1. Parasternal short-axis view at the level of the aortic valve (arrow) from an Echo in Africa study participant, demonstrating a bicuspid aortic valve with right-left coronary cusp fusion.

typically occur in later life, so differences in access to healthcare are unlikely to explain the differences observed in the present study. Finally, from the available literature, BAV does not appear to exhibit a predilection for either sex, so that the small sex difference observed in the different populations is unlikely to explain the marked difference in prevalence observed here. We therefore postulate that our black and mixed-ancestry populations have either an underlying protective trait against BAV or a relative absence of the causative genes.

This hypothesis is strengthened by the fact that our cohort demonstrated a gradient between black, mixed and white SA children. A prospective population study looking at the prevalence of congenital valve disease and how this relates to genetic and/or environmental factors is now required.

Funding. The EIA project is funded by an 'Every Heartbeat Matters' grant from Edwards Lifesciences (\#35526255).

\section{D Hunter}

Division of Cardiology, Tygerberg Academic Hospital and Faculty of Medicine and Health Sciences, Stellenbosch University, Cape Town, South Africa

14834782@sun.ac.za

\section{G W Lloyd}

Echocardiography Laboratory, Barts Heart Centre, St Bartholomew's Hospital, London, UK; Institute of Cardiovascular Sciences, University College London, UK; and William Harvey Research Institute, Queen Mary University of London, UK

\section{J Monaghan \\ King's College Hospital, London, UK}

\section{A J K Pecoraro, A F Doubell, P G Herbst}

Division of Cardiology, Tygerberg Academic Hospital and Faculty of Medicine and Health Sciences, Stellenbosch University, Cape Town, South Africa

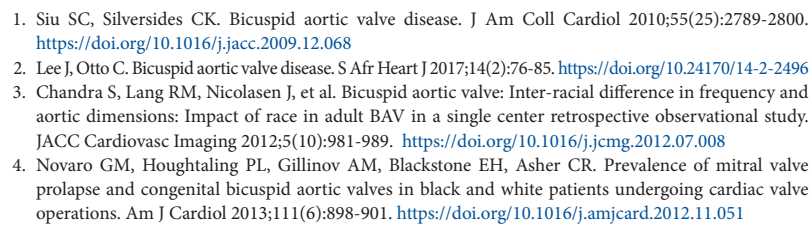

S Afr Med J 2020;110(8):706. https://doi.org/10.7196/SAMJ.2020.v110i8.14854 